\title{
Effects of errors under errorless and trial-and-error conditions
}

\author{
RONALD R. SCHMECK and EDDIE K. GROVE \\ Southern Ilinois University, Carbondale, Illinois 62901
}

\begin{abstract}
The authors employed a task which was actually composed of two subtasks performed in series. Error rate on the first subtask was manipulated while performance and retention on the second subtask were measured. Original learning of the second subtask was accomplished via an errorless procedure while the relearning session, held 1 week later, was conducted in trial-and-error fashion. Three levels of error were imposed on the subjects during original learning, and these were factorially combined with the same three levels during the relearning session. Imposed errors on the first subtask interfered with performance on the second subtask both during the original errorless learning session and during the trial-and-error relearning session. The imposed error rate had no effect on retention. The major performance deterioration occurred in the group that learned in the presence of a low error rate and relearned in the presence of a high error rate. The results were interpreted in terms of frustration theory.
\end{abstract}

Working within the theoretical framework that has come to be known as "frustration theory" (Amsel, 1962), Schmeck (1970) assumed that errors, since they impede goal-oriented progress, would produce a "frustration reaction" which raises the subject's drive level and disrupts subsequent task performance. The author conducted two experiments which employed a time-tested technique (e.g., Amsel \& Roussel, 1952) for demonstrating the presence of a motivational process. Specifically, both studies involved the use of two distinct subtasks performed in series on each trial. The presumably frustrating manipulation (errors) was repeatedly introduced on the first subtask, and its effects were repeatedly measured on the second subtask. A nonspecific motivational process was indicated, since an imposed increase in errors on the manipulated subtask produced an increase in errors on the measured subtask.

Unfortunately, Schmeck (1970) specifically instructed subjects to learn the correct responses to the manipulated subtask. Thus, they would have attempted to retain their experiences on the manipulated subtask from one trial to the next, i.e., while performing on the measured subtask. Furthermore, information load on the manipulated subtask within any given trial was greatest when a high error rate was imposed. This procedure failed to rule out the possibility that trial-to-trial retention of experiences on the manipulated subtask overloaded the memory of subjects in the high manipulated error group.

The present study attempted to rule out the memory-overload explanation by using a manipulated subtask which did not require trial-to-trial retention and which imposed an identical information load

This research was supported by grant No. 02-15-87 from the Southern Illinois University Office of Research and Projects. regardless of whether a response was indicated to be correct or incorrect. The study also examined the effect of manipulated subtask errors upon an errorless version of the measured subtask. Furthermore, assuming that a reduction in error-produced frustration might reinforce the learning of a correct response (cf. Daly, 1974), it was predicted that correct responses on the measured subtask would be "stronger" when finally acquired in the presence of a high error rate on the manipulated subtask. "Response strength" was operationally defined in terms of retention (relearning). The procedure also provided an opportunity to look for shift effects.

\section{METHOD}

\section{Subjects}

Subjects for the experiment were volunteers from introductory psychology classes at Southern Illinois University, each of whom participated in two sessions (original learning and relearning). Subjects were randomly assigned with the following exception. Twelve of the subjects were discarded for failure to meet the errorless criterion in original learning. Discarded subjects were replaced until there were 10 subjects in each of the nine groups (for a total of 90 subjects). No more than three subjects were discarded from any one experimental group. Subsequent interviews with these subjects indicated that virtually all of them had failed to fully understand the instructions.

\section{Design and Apparatus}

The separation of performance and retention effects was accomplished by means of a 3 by 3 factorial design with the factors being $30 \%, 50 \%$, and $70 \%$ error feedback on the manipulated subtask during original learning and during a relearning session 1 week later. In addition to providing an opportunity to separate performance and retention effects, this design also provided an opportunity to look for "shift effects" by comparing the performance of subjects trained under one error condition and then later retrained under a different error condition. In addition, the original training on the measured subtask was accomplished via an errorless procedure. If a high error rate on the manipulated subtask had raised the error rate on the measured subtask during original learning, this could have produced inferior retention (i.e., due to inferior original learning). 
It was hoped that this possibility could be eliminated by using an errorless procedure for the original learning of the measured subtask. Furthermore, this procedure provided an opportunity to observe the effects of error-produced frustration on the acquisition of an errorless task. Also, a postexperimental questionnaire was administered to gather information relevant to suspiciousness on the part of the subjects and to the manner in which they had divided their effort between the two subtasks.

The apparatus used was part of the Human Test System manufactured by Lehigh Valley Electronics. The manipulated subtask employed a 3 by 2 button matrix located in the lower center of the subject's console. The two buttons within each of the three rows differed on four stimulus dimensions: the location of the button (left vs. right); the symbol appearing on the face of the button (X vs. - ); the location of the symbol on the button (top vs. bottom); and the background color of the button (blue vs. yellow). Either button within any row was the same as either one in the previous row on two dimensions and different on two dimensions. The measured subtask employed a rear projection screen on which six different geometrical figures (the stimuli) could be presented. To the right of this module, there were three rear projection screens which presented the letters of CVC nonsense syllables (the responses). The subject's responses on this subtask were given orally and monitored via an intercom. Also, an add-subtract counter containing red and green lights was located immediately to the left of the button matrix. Correct responses and errors on both of the subtasks registered on this counter. The decision matrix and symbol-CVC subtasks were performed in series on each trial.

\section{Manipulated Subtask}

The decision matrix subtask required subjects to discover which of four stimulus dimensions was correct on each trial. To do this, the subject was to randomly choose one of the two buttons in the first row to serve as a "starting point." No feedback was given for this choice. He then chose one of the buttons in the second row. In all cases, this button was the same as the one chosen in the first row on two dimensions and was different on two dimensions. At this point, feedback was given, and regardless of whether the choice was indicated as correct or incorrect, the number of potentially "correct" dimensions was narrowed from four down to two. The subject then chose a button in the last row. The stimulus dimensions were arranged such that regardless of whether the choice was indicated to be correct or incorrect, the number of potentially "correct" dimensions was narrowed down to one. However, the subject's error rate throughout this set of decisions was, in fact, being manipulated by the experimenter. Since both the red and the green lights eliminated the same number of stimulus dimensions. there should have been no difference in information load on the manipulated subtask regardless of error rate. Furthermore, subjects were told that there would be absolutely no relationship between the stimulus dimension which was correct on one trial and the one which would be correct on the next trial. This eliminated any demand for trial-to-trial retention. It was specifically indicated that this subtask was designed to measure the subject's problem-solving ability and not his memory.

\section{Measured Subtask}

After the subject had responded to the third row of buttons on each decision matrix trial, he was presented with one of the six symbol-CVC pairs. This subtask was made errorless during original learning, and a trial-and-error procedure was used during relearning. The errorless procedure was similar to that employed by Touchette (1971). Specifically, a geometrical figure appeared in the first rear-projection screen, and the first letter of the CVC appeared $3.25 \mathrm{sec}$ later (the anticipation interval). Succeeding letters appeared at intervals of $1.25 \mathrm{sec}$. Subjects were instructed to learn the appropriate CVC response to each geometrical figure but to do so without error by never responding until they had seen enough letters to be absolutely confident that their response was correct. If they had any doubt whatsoever regarding the correct response, they were supposed to wait for the appearance of additional letters, even if they had to wait for the entire nonsense syllable to be presented. A response during the $3.25 \mathrm{sec}$ preceding the appearance of the first CVC letter indicated that they had thoroughly learned that response.

It was suspected that the groups subjected to the high manipulated error rate during original learning would require more trials to learn the measured subtask. Thus, a group of pilot subjects was run in the high error condition to determine the average number of trials required to learn the measured subtask. Since the pilot subjects required 25 trials, it was decided that all subjects in this study would be run until they were able to correctly anticipate the responses to all six symbols through two consecutive series of items (12 consecutive correct responses) or until they had been run for 25 trials, whichever came last. This was intended to equate average number of acquisition trials for all groups.

The relearning session was held 1 week after original learning. The procedure for the measured subtask was changed to trial and error. The interval between the presentation of the geometrical figure and the CVC response was $3.25 \mathrm{sec}$, but the three letters of the response were presented as a unit, and subjects were told to feel free to guess at the response during the anticipation interval. Subjects were run until they were able to correctly anticipate the responses to all six symbols through two consecutive series of items (12 consecutive correct responses). At the end of the relearning session, the subjects were given a questionnaire.

\section{RESULTS AND DISCUSSION}

\section{Original Learning}

The analysis of the errorless original learning data was performed on mean trials to last error. The experimenters determined, for each item for each subject, the last trial on which the subject did not anticipate the correct CVC. These scores were then averaged across all six items for each subject. The ANOVA indicated that the groups were significantly different $[F(2,87)=3.83, \quad p<.05] .^{1} \quad$ Subsequent analyses (Newman-Keuls) indicated that the low group $(M=19.87)$ and the medium group $(M=21.18)$ were not significantly different, but the high group $(M=27.07)$ differed significantly from both the low and the medium groups $(g<.05)$. Since there were no overt errors made during the original learning of the measured subtask, this finding suggests that errors not nnly interfere with the performance of what has been learned but may also interfere with the learning process itself, perhaps by disrupting attention (cf. Stoddard \& Sidman, 1967).

\section{Relearning}

Analysis of the trial-and-error relearning data again employed the measure of mean trials to last error calculated as described above. ${ }^{2}$ ANOVA indicated that the main effect for imposed error rate during original learning (the retention effect) was not significant, but the main effect for relearning error rate (the performance effect) was significant $[F(2,81)=4.37, p<.05]$. In addition, the interaction of the original learning and relearning error rates was significant $[\mathrm{F}(4.81)=3.53, \mathrm{p}<.05] .^{3}$

Inspection of Figure 1 suggested that both the 


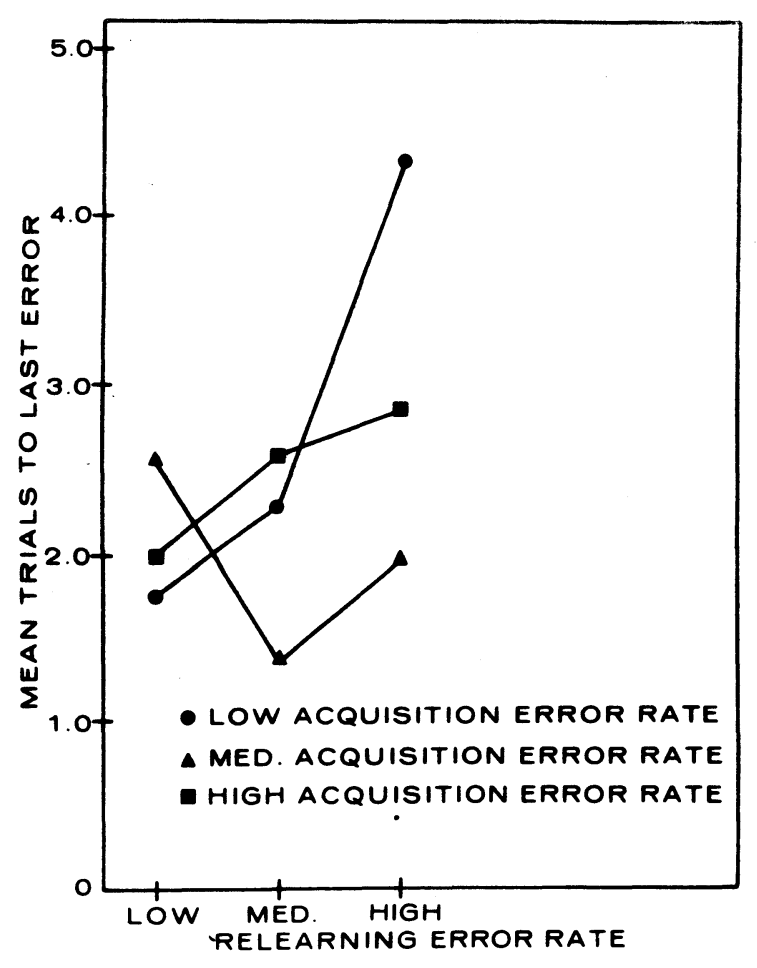

Figure 1. Mean trials to last error as a function of imposed error rate during original learning and during relearning.

performance and interaction effects might be attributable to the single data point for the low original/high relearning error rate group. Two statistical tests were conducted to test this hypothesis. First, an ANOVA demonstrated that the eight data points excluding the deviant point were not different $[F(7,82)=1.37]$. The second test, an orthogonal contrast of means, showed that the data point for the low original learning/high relearning error rate group is different from the other eight data points $[F(1,81)=19.84, p<.001]$. Thus, it appears that the performance disruption obtained during the relearning session was due to the particularly bad performance of the group that originally learned in the presence of the low and relearned in the presence of the high manipulated error rate. This suggests the presence of a contrast effect with subjects who had learned in a situation which was virtually errorless showing an extreme deterioration in performance when later tested in the presence of a high error rate (cf. Stoddard \& Sidman, 1967).

In regard to the postexperiment questionnaire, there were 12 questions relating in one way or another to suspiciousness on the part of the subjects and to the manner in which they had divided their effort between the two subtasks. In regard to suspiciousness, there was absolutely no indication in the answers to any of the questions that subjects had regarded the feedback as inaccurate or contrived. Also, $70 \%$ of the subjects who received the high manipulated error rate during both original learning and relearning specifically commented that they had found the task to be "frustrating." The comparable figure for subjects receiving low manipulated error rates was $25 \%$. The questions regarding effort division were aimed at determining whether subjects in the groups that had been subjected to the high manipulated error rate might have concentrated on the manipulated subtask to the exclusion of the measured subtask. This was apparently not the case since there were no differences between the groups in their reactions to any of these questions.

\section{REFERENCES}

AMSEL, A. Frustrative nonreward in partial reinforcement and discrimination learning; Some recent history and a theoretical extension. Psychological Review, 1962, 69, 306-328.

AMSEL, A., \& Roussel, J. Motivational properties of frustration: I. The effect on a running response of the addition of frustration to the motivational complex. Journal of Experimental Psychology, 1952, 43, 363-368.

DALY, H. B. Reinforcing properties of escape from frustration aroused in various learning situations. In G. H. Bower (Ed.), The psychology of learning and motivation (Vol. 8). New York: Academic Press, 1974.

SCHMECK, R. R. Error-produced frustration as a factor influencing the probability of occurrence of further errors. Journal of Experimental Psychology, 1970, 86, 153-156.

Stoddard, L. T., \& Sidman, M. The effects of errors on children's performance on a circle-elipse discrimination. Journal of the Experimental Analysis of Behavior, 1967, 10 , 261-270.

Touchette, P. E. Transfer of stimulus control: Measuring the moment of transfer. Journal of the Experimental Analysis of Behavior, 1971, 15, 347-354.

\section{NOTES}

1. In regard to variability, the $\mathrm{F}$ ratio calculated in this analysis was $440.92 / 115.20$.

2. Since the analysis of first trial recall scores and mean trials to last error produced the same results, only the latter analysis will be discussed.

3. Variability is indicated by the $F$ ratios: Original learning = $5.23 / 2.09$; relearning $=9.14 / 2.09 ;$ original by relearning $=7.39 / 2.09$.

(Received for publication September 29, 1975.) 